\title{
Systems Astrochemistry: A New Doctrine for Experimental Studies
}

\section{OPEN ACCESS}

Edited by:

Boutheïna Kerkeni,

Manouba University, Tunisia

Reviewed by:

Danna Qasim

National Aeronautics and Space

Administration, United States

Bhalamurugan Sivaraman,

Physical Research Laboratory, India

Christopher Bennett,

University of Central Florida,

United States

Albert Rimola,

Universitat Autònoma de Barcelona,

Spain

*Correspondence:

Nigel J. Mason

n.j.mason@kent.ac.uk

Perry A. Hailey

pah35@kent.ac.uk

Duncan V. Mifsud

duncanvmifsud@gmail.com

tORCID:

Nigel J. Mason

orcid.org/0000-0002-4468-8324;

Perry A. Hailey

orcid.org/0000-0002-8121-9674;

Duncan V. Mifsud

orcid.org/0000-0002-0379-354X;

James S. Urquhart

orcid.org/0000-0002-1605-8050

Specialty section:

This article was submitted to

Astrochemistry,

a section of the journal

Frontiers in Astronomy and Space

Sciences

Received: 09 July 2021

Accepted: 22 November 2021

Published: 08 December 2021

Citation:

Mason NJ, Hailey PA, Mifsud DV and Urquhart JS (2021) Systems Astrochemistry: A New Doctrine for Experimental Studies.

Front. Astron. Space Sci. 8:739046. doi: $10.3389 /$ fspas.2021.739046

\author{
Nigel J. Mason ${ }^{1 * t}$, Perry A. Hailey ${ }^{1 * t}$, Duncan V. Mifsud ${ }^{1,2 * t}$ and James S. Urquhart ${ }^{1 \dagger}$ \\ ${ }^{1}$ Centre for Astrophysics and Planetary Science, School of Physical Sciences, University of Kent, Canterbury, United Kingdom, \\ ${ }^{2}$ Institute for Nuclear Research (Atomki), Debrecen, Hungary
}

Laboratory experiments play a key role in deciphering the chemistry of the interstellar medium (ISM) and the formation of complex organic molecules (COMs) relevant to life. To date, however, most studies in experimental astrochemistry have made use of a reductionist approach to experimental design in which chemical responses to variations in a single parameter are investigated while all other parameters are held constant. Although such work does afford insight into the chemistry of the ISM, it is likely that several important points (e.g., the possible influence of experimental parameter interaction) remain ambiguous. In light of this, we propose the adoption of a new "systems astrochemistry" approach for experimental studies and present the basic tenants and advantages of this approach in this perspective article. Such an approach has already been used for some time now and to great effect in the field of prebiotic chemistry, and so we anticipate that its application to experimental astrochemistry will uncover new data hitherto unknown which could aid in better linking laboratory work to observations and models.

Keywords: astrochemistry, interstellar chemistry, molecular astrophysics, systems science, systems astrochemistry, design of experiments, complex systems

\section{INTRODUCTION}

One of the unexpected findings arising from the development of radio astronomy in the 1930s was the discovery of molecules in the interstellar medium (ISM), since until then all spectroscopic signatures had been atomic in nature. The confirmed presence of diatomic radicals such as $\mathrm{CN}$, CH, and OH (Swings and Rosenfeld, 1937, McKellar, 1940; Douglas and Herzberg, 1941; Weinreb et al., 1963) indicated that larger parent molecules should also exist in the ISM. ${ }^{1}$ This was subsequently confirmed through the discovery of formaldehyde (Snyder et al., 1969), and we are now aware of the interstellar presence of over 200 molecules including fullerenes, polycyclic aromatic hydrocarbons (PAHs), chiral structures, and biomolecules. ${ }^{2}$ A major challenge of astrochemistry (and the allied field of cosmochemistry) is to explain the formation of such a rich interstellar molecular inventory and, to this end, laboratory experiments which simulate conditions in the ISM have proven useful. Indeed, such experiments have also had implications for chemistry occurring within our own Solar System, so much so that the term "astrochemistry" is

\footnotetext{
${ }^{1}$ For a more complete description of the early history of astrochemistry, refer to the works of Feldman (2001) and Shematovich (2012).

${ }^{2}$ For a regularly updated list of molecules detected in the ISM, refer to the Cologne Database for Molecular Spectroscopy (https://cdms.astro.uni-koeln.de/classic/molecules).
} 
now taken to refer to chemistry occurring within any extraterrestrial environment, including the ISM, Solar System objects (particularly comets and icy moons), and exoplanets (Mumma and Charnley 2011; Caselli and Ceccarelli 2012; van Dishoeck 2014).

However, most of the experimental studies reported in the literature have made use of reductionist experimental designs, such as the "one-factor-at-a-time" (OFAT) approach in which a limited number of experimental variables or parameters are investigated by analyzing the effect of their individual variation on the resultant chemistry while all other parameters are kept constant (Czitrom 1999). This work has undoubtedly contributed to our knowledge of extra-terrestrial chemistry, but such an approach does mean that the influence of several (potentially key) variables on the outcome of the experiment is not observed. Moreover, the relationships between different experimental parameters cannot be comprehensively investigated using an OFAT approach. To compound matters further, a lack of standardization of equipment and techniques across the discipline has meant that it is likely that no two experiments truly replicate the results of the other and, indeed, certain assumptions made during experimentation mean that experimental conditions are not really representative of conditions in the ISM.

For example, the morphology of an ice is dependent upon that of the substrate to which it is adsorbed (Trakhtenburg et al., 1997). In the ISM, ices are adsorbed to carbonaceous or silicate dust grains with a highly irregular morphology containing steps, pores, cracks, and terraces (Dulieu et al., 2013; van Dishoeck 2014). This highly irregular morphology is thought to aid in the formation of astrochemical species through surface-catalyzed processes (Mendoza et al., 2004; Potapov et al., 2019; Suhasaria and Mennella 2021). In laboratory investigations of the chemistry occurring within interstellar ices, however, ices are usually deposited onto flat surfaces used for transmission or reflection spectroscopy (e.g., gold, zinc selenide, magnesium fluoride, etc.). Although previous studies have acknowledged that adsorbent morphology may play an important role in the chemistry or spectroscopy of the adsorbate ice (Perets et al., 2007; Gull et al., 2015; Qasim et al., 2017; Wakelam et al., 2017; Pantaleone et al., 2021), there is a scarcity of studies that have considered this experimentally, and fewer still that have considered the chemical influence of the interaction of flat substrates with incident radiation. One study by Mason et al. (2008) showed that the infrared spectrum of hexagonal crystalline water ice deposited over soot particles suspended in an ultrasonic trap differed somewhat to that of the same ice deposited onto flat fluoride substrates traditionally used in laboratory astrochemistry. This is significant, as it is believed that soot particles produced during combustion are morphologically similar to carbonaceous grains in the ISM (Cataldo and Pontier-Johnson 2002).

Understanding the influence of various parameters and parameter combinations, some of which may often be underreported or ignored in contemporary studies, is pivotal if laboratory astrochemistry is to accurately elucidate the conditions necessary for the formation of interstellar complex organic molecules $(\mathrm{COMs})^{3}$ and the mechanisms by which this occurs. However, in order to achieve such an exhaustive characterization of the molecular potential of different environments within the ISM, as well as different phases of stellar and planetary evolution, a new approach to experimental design and conduct is required. This new approach would carefully analyze the dependence of molecular synthesis on each parameter of the experiment, as well as parameter interactions. Such a multi-parameter approach requires a formal statistical design of the astrochemical experiment, and should thus adopt a "systems" approach. In systems chemistry, the focus is not on individual chemical components but rather on the overall network of interacting molecules and emergent properties (Ludlow and Otto 2008). The use of a systems approach also allows for the accumulation of coherent data sets which may be easily cross-correlated. In the rest of this article, we discuss the concepts of systems chemistry before elucidating how a "systems astrochemistry" approach may be developed so as to further our understanding of chemistry in the ISM and the formation of COMs with prebiotic significance. We note that the majority of our discussion will be dedicated to solid-phase experimental astrochemistry, as COMs in the ISM are thought to primarily form within icy grain mantles (Caselli and Ceccarelli 2012; van Dishoeck 2014) However, the underlying ideas and principles of our discussion could be applied equally well to gas-phase studies.

\section{SYSTEMS CHEMISTRY}

A complex system is defined as a collection of interdependent components capable of interacting with each other which is difficult to model due to the number and magnitude of competing interactions and relationships (Siegenfeld and BarYam 2020). The study of complex systems typically makes use of a holistic, systems-wide approach in which the focus is placed on the emergent properties of the system as a whole, rather than its constituent parts and their simple interactions (Whitesides and Ismagilov 1999; Ross and Arkin 2009). Complex systems analysis has been adopted in a number of scientific fields, the most relevant to this article is that of chemistry for which a "systems chemistry" paradigm has emerged (Ludlow and Otto 2008).

Systems chemistry frameworks seek to consider multiple variables and parameters simultaneously and focus on the emergent chemical products deriving from the complex system under investigation (Ludlow and Otto 2008; Li et al., 2013; Mattia and Otto 2015). This is in contrast to the reductionist approaches which have traditionally sought to understand bond formation from a simple, linear perspective, and which became widespread

${ }^{3}$ In astrochemistry, a complex organic molecule is typically defined as a molecule or molecular ion possessing six or more constituent atoms. Although this definition may be utilized to equal effect in the discussion presented in this article, our focus is more centred around large molecules which may have prebiotic relevance (e.g., amino acids, nucleic acids, carbon chains). 
due to limitations in the available analytical equipment and methodologies, specific requirements related to reactions yields and product purities, and the consensus that understanding smaller and simpler components of a system may provide some insight into more complex chemistry.

Although still in its infancy, a systems chemistry framework has already been adopted in the field of prebiotic chemistry to study the assembly of chemical sub-systems into an overall larger system with a focus on its emergent physico-chemical properties (Powner and Sutherland 2011). This has led to a fundamental change in our approach to understanding the chemistry of life's origins by considering molecules beyond those that are used by extant biology, including prebiotically relevant molecules that existed alongside biogenic ones (Krishnamurthy 2020). Results from such prebiotic systems chemistry work have been farreaching, with the emergence of RNA being demonstrated to not be a simple outcome of reactions between plausible prebiotic precursors but rather a systems chemistry emergence from a library of molecules (Kim et al., 2017). Additionally, problems related to prebiotic ribonucleotide selection have been shown to be circumvented by a system-wide sequestration of glyceraldehyde by 2-aminothiazole (Islam et al., 2017; Islam and Powner 2017).

If adapted to suit laboratory research in astrochemistry, a systems chemistry approach would allow for the effects of multiple experimental parameters and variables to be considered and quantified, which may yield significant insights into the formation of COMs. As discussed above, laboratory astrochemistry as it has been practiced thus far has largely adopted an OFAT approach centered either on simple ices irradiated by a single processing type (e.g., ion beams or ultraviolet photons), or on the formation of complex materials via the irradiation of mixed ices and substrates. We refer the interested reader to the works of Allodi et al. (2013), Linnartz et al. (2015), Öberg (2016), and Arumainayagam et al. (2019) for reviews on the current state-of-the-art of laboratory astrochemistry. The challenge thus is to learn from such a working paradigm to develop a coherent understanding of the formation of COMs in a structured yet relevant manner. Indeed, the need for a more systematic approach has already been referred to in a number of publications (e.g., Carota et al., 2015; Gentili 2020; James et al., 2020).

We therefore propose that a systems astrochemistry framework be adopted in laboratory astrochemistry experiments, wherein multiple variables and parameters are studied simultaneously under conditions relevant to the ISM. That is to say, the physico-chemical constraints, role of ice morphology and polarity, grain catalysis, multiple processing methods, and the recycling of molecular material during interstellar cloud evolution should all be simultaneously studied in a structured systematic design to produce the conditions under which the chemical inheritance of COM formation is explored. In Figure 1, three levels of investigation are proposed. Level 1 refers to the chemical space, which is the space of all possible molecules (including organic, inorganic, and element species) and from which chemical networks will emerge when processed by Level 2. Level 2 is the environmental space and

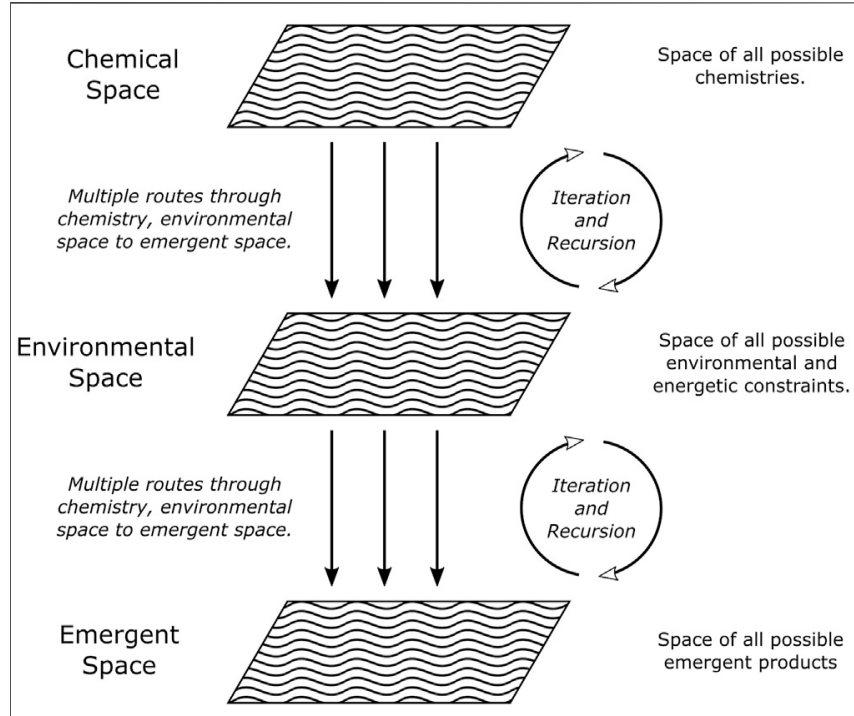

FIGURE 1 | Graphical representation of the systems astrochemistry concept highlighting the different levels of adaptation.

represents the energetic, environmental, heterogenic, and physical constraints: this layer would provide the "driving force" behind state transitions to organized emergent products. Finally, Level 3 is the emergent space wherein COMs and the transitions from non-living to living matter could occur. Figure 1 also attempts to demonstrate that the process is not necessarily linear and processing of the chemical space by the environmental space is likely to be iterative and recursive. The remainder of this section is devoted to discussing the themes relevant to a proposed systems astrochemistry experiment.

\section{Chemical Space}

During star formation, interstellar icy grain mantles are subjected to changing physico-chemical conditions and reprocessing environments which lead to increased molecular complexity. Presently, the vast majority of laboratory astrochemistry experiments seeking to understand these processes make use of a single method of ice processing and, as such, only the chemical outcomes of very specific circumstances are deciphered. However, in order to obtain a more realistic view of interstellar chemistry, sequential irradiation of different processing types, co-irradiation of different processing types, and cycles of heating, desorption, and cooling also need to be built into the experimental design. Understanding the influence of such changing parameters is crucial, as it is already known that different processing methods result in different chemistry, and may thus produce a different chemical feedstock for subsequent reactions (Mullikin et al., 2018).

When adopting a systems astrochemistry approach, the concept of a chemical space must be considered. The formation of molecules in the ISM involves both simple and complex molecules and, given that COM formation is not necessarily a linear or orthogonal process, chemical formation in the ISM resulting from component chemistries should be 
explored so as to further develop an understanding of the astrochemical pathways. This inventory of molecules provides a source for molecular assembly which may be used to develop our understanding of competing and complementary astrochemical pathways during molecule formation in the ISM. To illustrate this point, the simple energetic processing of a single-component or two-component ice is known to generate a wealth of molecules (e.g., Henderson and Gudipati 2015). This pool of molecules is then capable of undergoing further energetic processing to produce different, more complex molecules. This example demonstrates the vast array of molecules which could be formed from relatively simple starting ices. As such, applying combinatorial or molecular assembly strategies for the selection of libraries of molecules in a systematic manner could reveal interesting chemistries. A similar approach has been demonstrated recently in a prebiotic chemistry experiment wherein a fully automated system explored complex mixtures over extended time-frames, all the while taking a systems view of the multi-component chemistry (Asche et al., 2021).

\section{Environmental Space}

This space should look to mimic the conditions in the ISM, considering all relevant factors such as the temperature(s) under investigation, the energetic processing type (e.g., ion and/or photon irradiation), the substrate (including its composition and morphology), the pressure of the environment, the polarity, morphology, and chemical composition of the ice analogue, the time for various factors including deposition and processing, the type of chemistry occurring (e.g., photochemistry, electron-induced chemistry), and any other relevant physical and chemical constraints. The environmental space acting upon the chemical space results in the emergent properties that are the result of controlled processing of molecules and substrates under ISM relevant conditions. This can be achieved by considering all the relevant factors listed in the environmental space and how they process a constrained chemical space.

Laboratory astrochemistry experiments have typically employed an OFAT approach in which one factor is varied while all the others are held constant. Such experiments are useful as they are simple to execute and comparatively quick to perform. However, a major disadvantage of studies using an OFAT approach is that the relationships between experimental factors cannot be investigated. Instead, we encourage laboratory experimentalists to embrace formal designs of experiments (DoEs) as part of a systems astrochemistry approach. There exists a variety of formal DoEs and which one to select depends upon the overall experimental goal and constraints; however, typical designs include screening, response surface, Taguchi Array, mixture, and split plot designs. Selecting the correct DoE allows all relevant factors to be built into the experiment and varied in a series of experimental runs, after which the outcome from each run is fitted against the product responses (i.e., the emergent space). Formal DoEs may require more experiments to be performed than an OFAT approach but benefit from revealing the interactions between variable factors and their impact on the emergent properties of the system that could otherwise be misinterpreted using an OFAT approach. A more complete explanation of the use of DoEs in chemical studies may be found in the work of Deming and Morgan (1993).

\section{Emergent Space}

By embracing the complexity of the chemical and environmental space, the emergent space gives rise to new and interesting chemistry that provides information into the underlying components and their interactions. In laboratory astrochemistry terms, the emergent properties from a welldesigned, multi-component and multi-factor experiment will shed light on the properties of chemical recycling and inheritance (e.g., in COM formation in a protostar). To unravel this complexity requires the adoption of parallel and multiplexed screening analytical technologies with the application of machine learning approaches for data decomposition and feature extraction.

\section{Analytical and Processing Considerations}

In laboratory astrochemistry, the most commonly used in situ analytical techniques are quadrupole mass spectrometry (QMS) and Fourier-transform mid-infrared spectroscopy (FTIR). Other techniques are also becoming more popular: the use of millimeter/sub-millimeter and terahertz spectroscopy, for instance, will likely help bridge results from the laboratory to observational studies (e.g., Doménech et al., 2017; Chantzos et al., 2019; Widicus Weaver 2019; Yocum et al., 2019; Zakharenko et al., 2019; Bizzocchi et al., 2020; Stahl et al., 2020; Mifsud et al., 2021a), while in situ transmission electron microscopy (TEM) has recently been used to great effect in understanding diffusion and crystallization in ices (Kouchi et al., 2020; Tsuge et al., 2020; Kouchi et al., 2021). Although in situ techniques are useful in identifying functional groups present in the ice and sputtered or desorbed molecules in the gas phase, some of the products form a refractory solid residue which is too complex for its individual components to be resolved via FTIR spectroscopy.

As such, much of the complex chemistry is hidden from in situ analysis. For this reason, ex situ analysis of residues is becoming increasingly popular (e.g., Nuevo et al., 2012; Materese et al., 2017; Materese et al., 2018). This has coincided with progress in analytical methodologies which have allowed greater sensitivities to be accessed with the advent of hyphenated and multidimensional techniques such as GC-MS/MS, LC-MS/MS, parallel chromatographic techniques using multiplexed diode array detectors (DADs), and improved mass spectrometry. Ex situ analysis does present some challenges due to the need to remove the sample from the astrochemical chamber and secure its transportation, all the while being sure to preclude unwanted physico-chemical changes to the residue (e.g., oxidation due to contact with ambient air). A properly validated analytical methodology should thus account for these factors (Fulvio et al., 2021).

Astrochemical phenomena may be viewed as a combination of processes occurring on an event-by-event basis, making sequential or parallel processing studies using processing methods of different types a potentially interesting route for laboratory studies. However, if such an investigation is to be performed, then it is necessary for multiple energetic sources (e.g., 
ion beamlines, electron guns, ultraviolet lamps) to be available at the same research facility. The "complete" astrochemistry experiment would thus have access to a range of processing types, including ion and electron sources with wide energy ranges alongside vacuum- and broadband ultraviolet photon sources. Other processing types, such as gamma and X-rays, could also be included.

This is, of course, an idealized situation, and it is highly unlikely that a single laboratory facility could host all such radiation sources. However, some experimental astrochemistry groups have been successful in incorporating multiple energetic sources into a single set-up (e.g., Herczku et al., 2021; Mifsud et al., 2021b), while others have made their chambers portable, allowing for them to be transported to different facilities offering different processing types (e.g., Ioppolo et al., 2020). Such workarounds may be the most cost-effective and technically feasible ways of incorporating multiple processing types into a statistical experimental design.

\section{Workflow Automation and Intelligent Control}

Adopting systems astrochemistry will require a holistic, integrated, modular, and a more standardized approach to the automation and control of the instrumentation and components of the laboratory astrochemistry system. This will provide improvements in repeatability, reproducibility, automation of experimental workflows, data storage and access, secure remote access, and, through the digital capture of the workflow, allow the transfer of "digital" experiments to other facilities adopting a similar standardized approach. The challenge described here is one that has seen significant success in the manufacturing sector where standards such as ISA S95 and ISA S88 have been adopted.

ISA S95 provides a framework for defining architectural abstraction layers in a system and their function, while ISA S88 provides the framework for defining equipment control, procedural workflows, and recipes. Adopting such an approach for laboratory astrochemistry could be achieved using a supervisory control and data acquisition (SCADA) system architecture. This would provide fully automated workflows via text-based entry, orchestration of instrumentation and equipment, data acquisition, consolidation, and storage into a historian ${ }^{4}$ via a single human-machine interface. Additionally, such an architecture could be opened up to secure remote access and would provide for standardization and interoperability of experiments between facilities (Figure 2). Learning from industry and adopting similar approaches in systems astrochemistry will accelerate the development and use of fully automated systems.

${ }^{4}$ The term "historian" is used extensively in industry and refers to the data repository for all operational and experimental data aggregation and storage.

\section{Data Analysis}

The completed systems astrochemistry experiment will generate a significant amount of data and so new approaches to data analysis are required which will differ greatly to the univariate approach currently used by mainstream astrochemistry studies. The adaption of multi-variate techniques from the fields of chemometrics, machine learning, and observational astronomy may reveal insights into data hitherto unmined. It should be noted that there do exist some published attempts to extract information from higher dimensional data sets using van Krevelen diagrams or high-resolution FTIR and mass spectrometric data (e.g., Wollrab et al., 2016; Ruf et al., 2018). However, although such attempts do provide more insight into the chemical similarity of organic components and provide for excellent data visualization, they are still rather equivocal. The application of a screening approach to the analysis of higher dimensional data sets combined with feature extraction and data reduction techniques may reveal insights into this complex chemistry. Principle component analysis (PCA), for example, has been used in the field of observational astronomy where it has proven useful in revealing the relationship between the principal components and ionization state of observed PAHs (Sidhu et al., 2021).

The use of a control system to manage the aggregation of data from multiple sensors would allow these data sets to be explored using a wide variety of machine learning techniques. Machine learning is a broad, well-developed field and it is not the intent of this article to give a comprehensive overview of this topic, but rather to illustrate its potential applicability to systems astrochemistry. As such, a thorough description of all possible machine learning techniques which could be employed in data analysis goes beyond the scope of this work. The authors instead direct the interested reader to the work of Brunton and Kutz (2019) for a more detailed introduction to and discussion of machine learning. In the interest of including a few examples in this article, however, we have illustrated a few machine learning techniques which may possibly be applied to systems astrochemistry in Figure 3.

\section{Modelling in the Context of Systems Astrochemistry: Introducing the Digital Twin} Conclusions within astrochemistry are often reached by linking together the results obtained by the three major research activities: laboratory experiments, observational astronomy, and astrochemical modelling. Such an approach has provided great insight into understanding the formation of COMs in the ISM. However, in putting forward the idea of a systems astrochemistry framework, we further propose that an additional consideration be made such that the experimentation occurring within laboratory astrochemistry chambers is also modelled via the use of a digital twin.

A digital twin is a virtual representation of a physical system that serves as an in silico replica for scenario planning, sensitivity analysis, and modelling of responses to perturbations or changes in the chemical or environmental spaces. The concept of a digital twin was first adopted in 2010 by NASA in an attempt to improve 


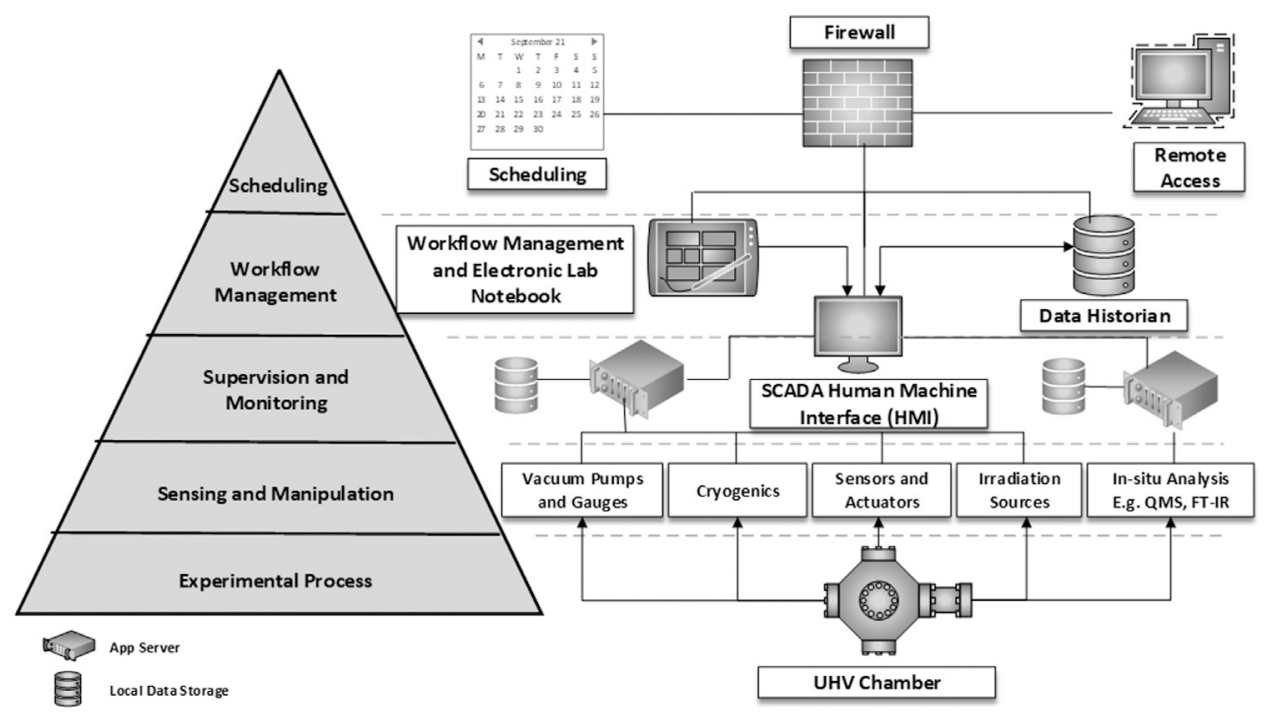

FIGURE 2 | Conceptual ISA S95 designed SCADA system architecture of the type proposed to be incorporated into systems astrochemistry experiments.

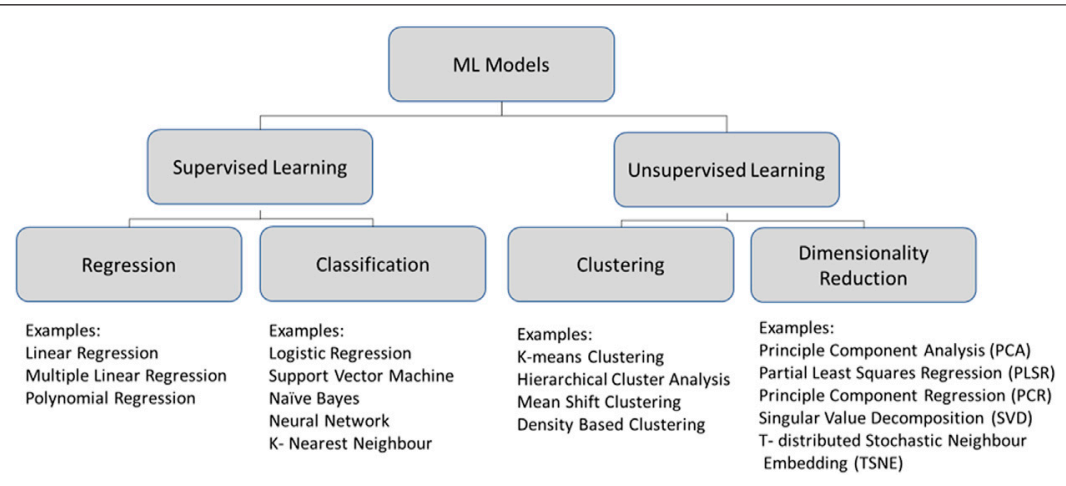

FIGURE 3 | Simple guide to the machine learning (ML) approach which we propose be applied to systems astrochemistry.

spacecraft simulation (Negri 2017) and has since been adopted widely within industrial settings, especially in manufacturing plants where digital twins support the digital transformation and adoption of Industry 4.0 concepts and model production efficiency. Industry is adopting the digital twin approach to enable in silico scenario planning of the changes to manufacturing routines and their impact upon overall equipment effectiveness, investigations into the impact of parameter changes of line automation and equipment on product quality, and plant efficiency.

Developing a digital twin for a laboratory astrochemistry experiment operating under a systems astrochemistry approach would accrue significant scientific benefits, particularly in the areas of sensitivity analysis and scenario planning of changes to experimental parameters and their impact on the emergent chemistry. Thus, when underpinned by statistically constrained DoEs, changes in chemical and environmental spaces may be successfully modelled. Examples of such modelling might include assessing the sensitivity of emergent chemical properties to changes in energetic parameters, or the in silico testing of experiments prior to experimental runs (which would be very useful to research groups with limited access to central research facilities).

\section{Linking Astrochemistry and Cosmochemistry: The Use of Isotopes}

An intimate link exists between the fields of astrochemistry and prebiotic chemistry, given that they are both concerned with the formation of COMs relevant to biology, albeit under different chemical and environmental spaces. Indeed, our suggested adaption and adoption of the systems approach used in prebiotic chemistry to astrochemical experiments highlights this link. One area of research which perhaps has not yet been fully exploited is the role of isotopes in the study of astrochemical reactions, particularly those occurring in interstellar ice analogues. 
Contemporary work has largely used isotopes to unravel details related to reaction mechanisms (Jamieson and Kaiser 2007; Bennett et al., 2010; Lamberts et al., 2017), and such work should indeed constitute a part of systems astrochemistry studies so as to further elucidate the dominant reaction pathways associated with different experimental or astrophysical conditions. The consideration of isotopes as an experimental factor in systems astrochemistry, however, also presents an opportunity to bridge the gap to the related field of cosmochemistry, where isotope fractionations associated with processing undergone by minerals, residues, and refractory materials are considered. Such fractionations may also occur in ices as a result of photochemistry or radiation chemistry, however experimental studies looking into such possible isotope enrichments (e.g., Charnley and Rodgers 2002; Sandford et al., 2010; Vinogradoff et al., 2013; Sugahara et al., 2019) are uncommon.

The systems analysis of isotope fractionations in the solid phase is thus recommended, as it would not only expand our knowledge of astrochemical processes in the ISM, but also provide further insight to the formation and composition of the molecular and mineral building blocks of Solar Systems. By adapting existing analytical techniques currently used in isotope geochemistry (such as combined element analysis and isotope ratio mass spectrometry) and incorporating them into an experimental systems astrochemistry framework, additional and hitherto unknown tracers for the processing history of various celestial solid objects, including comets, Kuiper Belt Objects, meteorites, and interplanetary dust, could be discovered.

\section{CONCLUSION}

In this article, we have discussed the core principles of a proposed new approach to laboratory astrochemistry based on systems chemistry which involves the use of formal DoEs that consider all relevant experimental factors simultaneously. Such an approach is advantageous when compared to the traditionally used OFAT approach, as a greater insight may be gleaned into the influence of such parameters on the emergent properties of the system, as well as the possible interaction between different parameters. Additionally, the adoption of this systems astrochemistry approach would pave the way for equipment and methodology

\section{REFERENCES}

Allodi, M. A., Baragiola, R. A., Baratta, G. A., Barucci, M. A., Blake, G. A., Boduch, P., et al. (2013). Complementary and Emerging Techniques for Astrophysical Ices Processed in the Laboratory. Space Sci. Rev. 180, 101-175. doi:10.1007/ s11214-013-0020-8

Arumainayagam, C. R., Garrod, R. T., Boyer, M. C., Hay, A. K., Bao, S. T., Campbell, J. S., et al. (2019). Extraterrestrial Prebiotic Molecules: photochemistryvs.Radiation Chemistry of Interstellar Ices. Chem. Soc. Rev. 48, 2293-2314. doi:10.1039/c7cs00443e

Asche, S., Cooper, G. J. T., Keenan, G., Mathis, C., and Cronin, L. (2021). A Robotic Prebiotic Chemist Probes Long Term Reactions of Complexifying Mixtures. Nat. Commun. 12, 3547. doi:10.1038/s41467-021-23828-Z standardization across the field, allowing for better crosscorrelation and reproduction of studies.

Astrochemistry is a mature scientific discipline which is entering an exciting age characterized by the deployment of new space- and ground-based telescopes and the commissioning and expansion of experimental facilities. We believe that the adoption of systems astrochemistry in laboratory experiments would allow for the maximum potential of these new facilities to be exploited, and thus greatly increase our understanding of the formation of COMs in the ISM and their influence on the emergence of life on Earth (and possibly elsewhere). Indeed, many of the topics discussed in this article have already been used to great effect in prebiotic chemistry research and in industrial settings, and we ourselves are currently engaged in establishing laboratory facilities and generating preliminary data based on a systems astrochemistry approach. It is therefore likely that the adoption of the systems astrochemistry approach proposed in this article would allow the astrochemistry research community to realistically answer some of the greatest remining questions of modern astronomy.

\section{DATA AVAILABILITY STATEMENT}

The original contributions presented in the study are included in the article/Supplementary Material, further inquiries can be directed to the corresponding authors.

\section{AUTHOR CONTRIBUTIONS}

All authors listed have made a substantial, direct, and intellectual contribution to the work and approved it for publication.

\section{FUNDING}

The authors are grateful to have received funding from the Europlanet 2024 RI which has been funded by the European Union Horizon 2020 Research Innovation Program under grant agreement No. 871149. DVM is the grateful recipient of a University of Kent Vice-Chancellor's Research Scholarship.

Bennett, C. J., Jamieson, C. S., and Kaiser, R. I. (2010). Mechanistical Studies on the Formation and Destruction of Carbon Monoxide (CO), Carbon Dioxide (CO2), and Carbon Trioxide (CO3) in Interstellar Ice Analog Samples. Phys. Chem. Chem. Phys. 12, 4032. doi:10.1039/b917162b

Bizzocchi, L., Prudenzano, D., Rivilla, V. M., Pietropolli-Charmet, A., Giuliano, B. M., Caselli, P., et al. (2020). Propargylimine in the Laboratory and in Space: Millimetre-Wave Spectroscopy and its First Detection in the ISM. A\&A 640, A98. doi:10.1051/0004-6361/202038083

Brunton, S. L., and Kutz, J. N. (2019). Data-Driven Science and Engineering Machine Learning, Dynamical Systems and Control. Cambridge University Press.

Carota, E., Botta, G., Rotelli, L., Di Mauro, E., and Saladino, R. (2015). Current Advances in Prebiotic Chemistry under Space Conditions. Curr. Org. Chem. 19, 1963-1979. doi:10.2174/1385272819666150622175143 
Caselli, P., and Ceccarelli, C. (2012). Our Astrochemical Heritage. Astron. Astrophys. Rev. 20, 56. doi:10.1007/s00159-012-0056-x

Cataldo, F., and Pontier-Johnson, M. A. (2002). Recent Discoveries in Carbon Black Formation and Morphology and Their Implications on the Structure of Interstellar Carbon Dust. Fullerenes, Nanotubes and Carbon Nanostructures 10, 1-14. doi:10.1081/fst-120002925

Chantzos, J., Spezzano, S., Endres, C., Bizzocchi, L., Lattanzi, V., Laas, J., et al. (2019). Rotational Spectroscopy of the HCCO and DCCO Radicals in the Millimeter and Submillimeter Range. A $\triangleleft A$ 621, A111. doi:10.1051/0004-6361/ 201834419

Charnley, S. B., and Rodgers, S. D. (2002). The End of Interstellar Chemistry as the Origin of Nitrogen in Comets and Meteorites. Astrophys. J. 569, L133-L137. doi: $10.1086 / 340484$

Czitrom, V. (1999). One-Factor-at-a-Time versus Designed Experiments. The Am. Statistician 53, 126. doi:10.2307/2685731

Deming, S. N., and Morgan, S. L. (1993). Experimental Design: A Chemometric Approach. Elsevier Science.

Doménech, J. L., Schlemmer, S., and Asvany, O. (2017). Accurate Frequency Determination of Vibration-Rotation and Rotational Transitions of $\mathrm{SiH}^{+}$. Astrophys. J. 849, 60. doi:10.3847/1538-4357/aa8fca

Douglas, A. E., and Herzberg, G. (1941). Note on $\mathrm{CH}\{+\}$ in Interstellar Space and in the Laboratory. ApJ 94, 381. doi:10.1086/144342

Dulieu, F., Congiu, E., Noble, J., Baouche, S., Chaabouni, H., Moudens, A., et al. (2013). How Micron-Sized Dust Particles Determine the Chemistry of Our Universe. Sci. Rep. 3, 1338. doi:10.1038/srep01338

Feldman, P. A. (2001). Molecular Astronomy from a Canadian Perspective: The Early Years. Can. J. Phys. 79, 89-100. doi:10.1139/p01-002

Fulvio, D., Potapov, A., He, J., and Henning, T. (2021). Astrochemical Pathways to Complex Organic and Prebiotic Molecules: Experimental Perspectives for In Situ Solid-State Studies. Life 11, 568. doi:10.3390/life11060568

Gentili, P. L. (2020). "Astrochemistry and the Theory of Complex Systems," in Proceedings of the Observatory for Astrochemical Kinetics and Related Aspects (Rome (Italy): Accademia delle Scienze).

Gull, M., Mojica, M. A., Fernández, F. M., Gaul, D. A., Orlando, T. M., Liotta, C. L., et al. (2015). Nucleoside Phosphorylation by the Mineral Schreibersite. Sci. Rep. 5, 17198. doi:10.1038/srep17198

Henderson, B. L., and Gudipati, M. S. (2015). Direct Detection of Complex Organic Products in Ultraviolet (Lya) and Electron-Irradiated Astrophysical and Cometary Ice Analogs Using Two-step Laser Ablation and Ionization Mass Spectrometry. ApJ 800, 66. doi:10.1088/0004-637x/800/1/66

Herczku, P., Mifsud, D. V., Ioppolo, S., Juhász, Z., Kaňuchová, Z., Kovács, S. T. S., et al. (2021). The Ice Chamber for Astrophysics-Astrochemistry (ICA): A New Experimental Facility for Ion Impact Studies of Astrophysical Ice Analogs. Rev. Scientific Instr. 92, 084501. doi:10.1063/5.0050930

Ioppolo, S., Kaňuchová, Z., James, R. L., Dawes, A., Jones, N. C., Hoffmann, S. V., et al. (2020). Vacuum Ultraviolet Photoabsorption Spectroscopy of SpaceRelated Ices: $1 \mathrm{keV}$ Electron Irradiation of Nitrogen- and Oxygen-Rich Ices. Astron. Astrophys. 641, 154. doi:10.1051/0004-6361/201935477

Islam, S., Bučar, D.-K., and Powner, M. W. (2017). Prebiotic Selection and Assembly of Proteinogenic Amino Acids and Natural Nucleotides from Complex Mixtures. Nat. Chem 9, 584-589. doi:10.1038/nchem.2703

Islam, S., and Powner, M. W. (2017). Prebiotic Systems Chemistry: Complexity Overcoming Clutter. Chem. 2, 470-501. doi:10.1016/j.chempr.2017.03.001

James, R. L., Ioppolo, S., Hoffmann, S. V., Jones, N. C., Mason, N. J., and Dawes, A. (2020). Systematic Investigation of CO2 : NH3 Ice Mixtures Using Mid-IR and VUV Spectroscopy - Part 1: thermal Processing. RSC Adv. 10, 37515-37528. doi:10.1039/d0ra05826b

Jamieson, C. S., and Kaiser, R. I. (2007). Isotopic Study of the Formation of the Azide Radical (N3). Chem. Phys. Lett. 440, 98-104. doi:10.1016/ j.cplett.2007.04.042

Kim, E.-K., Martin, V., and Krishnamurthy, R. (2017). Orotidine-Containing RNA: Implications for the Hierarchical Selection (Systems Chemistry Emergence) of RNA. Chem. Eur. J. 23, 12668-12675. doi:10.1002/chem.201702912

Kouchi, A., Furuya, K., Hama, T., Chigai, T., Kozasa, T., and Watanabe, N. (2020). Direct Measurements of Activation Energies for Surface Diffusion of CO and $\mathrm{CO} 2$ on Amorphous Solid Water Using In Situ Transmission Electron Microscopy. ApJ 891, L22. doi:10.3847/2041-8213/ab78a2
Kouchi, A., Tsuge, M., Hama, T., Oba, Y., Okuzumi, S., Sirono, S.-i., et al. (2021). Transmission Electron Microscopy Study of the Morphology of Ices Composed of $\mathrm{H} 2 \mathrm{O}, \mathrm{CO} 2$, and $\mathrm{CO}$ on Refractory Grains. ApJ 918, 45. doi:10.3847/15384357/ac0ae6

Krishnamurthy, R. J. (2020). Systems Chemistry in the Chemical Origins of Life: The $18^{\text {th }}$ Camel Paradigm. J. Syst. Chem. 8, 40.

Lamberts, T., Fedoseev, G., Kästner, J., Ioppolo, S., and Linnartz, H. (2017). Importance of Tunneling in $\mathrm{H}$-Abstraction Reactions by $\mathrm{OH}$ Radicals. A\&A 599, A132. doi:10.1051/0004-6361/201629845

Li, J., Nowak, P., and Otto, S. (2013). Dynamic Combinatorial Libraries: From Exploring Molecular Recognition to Systems Chemistry. J. Am. Chem. Soc. 135, 9222-9239. doi:10.1021/ja402586c

Linnartz, H., Ioppolo, S., and Fedoseev, G. (2015). Atom Addition Reactions in Interstellar Ice Analogues. Int. Rev. Phys. Chem. 34, 205-237. doi:10.1080/ 0144235x.2015.1046679

Ludlow, R. F., and Otto, S. (2008). Systems Chemistry. Chem. Soc. Rev. 37, 101-108. doi:10.1039/b611921m

Mason, N. J., Drage, E. A., Webb, S. M., Dawes, A., McPheat, R., and Hayes, G. (2008). The Spectroscopy and Chemical Dynamics of Microparticles Explored Using an Ultrasonic Trap. Faraday Discuss. 137, 367-376. doi:10.1039/ b702726p

Materese, C. K., Nuevo, M., McDowell, B. L., Buffo, C. E., and Sandford, S. A. (2018). The Photochemistry of Purine in Ice Analogs Relevant to Dense Interstellar Clouds. ApJ 864, 44. doi:10.3847/1538-4357/aad328

Materese, C. K., Nuevo, M., and Sandford, S. A. (2017). The Formation of Nucleobases from the Ultraviolet Photoirradiation of Purine in Simple Astrophysical Ice Analogues. Astrobiology 17, 761-770. doi:10.1089/ ast.2016.1613

Mattia, E., and Otto, S. (2015). Supramolecular Systems Chemistry. Nat. Nanotech 10, 111-119. doi:10.1038/nnano.2014.337

McKellar, A. (1940). Evidence for the Molecular Origin of Some Hitherto Unidentified Interstellar Lines. Publ. Astron. Soc. Pac. 52, 187. doi:10.1086/ 125159

Mendoza, C., Ruette, F., Martorell, G., and Rodrguez, L. S. (2004). Quantumchemical Modeling of Interstellar Grain Prebiotic Chemistry: Catalytic Synthesis of Glycine and Alanine on the Surface of a Polycyclic Aromatic Hydrocarbon Flake. ApJ 601, L59-L62. doi:10.1086/381800

Mifsud, D. V., Hailey, P. A., Traspas Muiña, A., Auriacombe, O., Mason, N. J., and Ioppolo, S. (2021a). The Role of Terahertz and Far-IR Spectroscopy in Understanding the Formation and Evolution of Interstellar Prebiotic Molecules. Front. Astron. Space Sci. 8, 757619. doi:10.3389/fspas.2021.757619

Mifsud, D. V., Juhász, Z., Herczku, P., Kovács, S. T. S., Ioppolo, S., Kaňuchová, Z., et al. (2021b). Electron Irradiation and thermal Chemistry Studies of Interstellar and Planetary Ice Analogues at the ICA Astrochemistry Facility. Eur. Phys. J. D 75, 182. doi:10.1140/epjd/s10053-021-00192-7

Mullikin, E., van Mulbregt, P., Perea, J., Kasule, M., Huang, J., Buffo, C., et al. (2018). Condensed-Phase Photochemistry in the Absence of Radiation Chemistry. ACS Earth Space Chem. 2, 863-868. doi:10.1021/ acsearthspacechem. $8 \mathrm{~b} 00027$

Mumma, M. J., and Charnley, S. B. (2011). The Chemical Composition of CometsEmerging Taxonomies and Natal Heritage. Annu. Rev. Astron. Astrophys. 49, 471-524. doi:10.1146/annurev-astro-081309-130811

Negri, E., Fumagalli, L., and Macchi, M. (2017). A Review of the Roles of Digital Twin in CPS-Based Production Systems. Proced. Manufacturing 11, 939-948. doi:10.1016/j.promfg.2017.07.198

Nuevo, M., Milam, S. N., and Sandford, S. A. (2012). Nucleobases and Prebiotic Molecules in Organic Residues Produced from the Ultraviolet PhotoIrradiation of Pyrimidine in NH3and $\mathrm{H} 2 \mathrm{O}+\mathrm{NH} 3 \mathrm{Ices}$. Astrobiology 12, 295-314. doi:10.1089/ast.2011.0726

Öberg, K. I. (2016). Photochemistry and Astrochemistry: Photochemical Pathways to Interstellar Complex Organic Molecules. Chem. Rev. 116, 9631. doi:10.1021/ acs.chemrev.5b00694

Pantaleone, S., Corno, M., Rimola, A., Balucani, N., and Ugliengo, P. (2021). Ab Initio Computational Study on Fe2NiP Schreibersite: Bulk and Surface Characterization. ACS Earth Space Chem. 5, 1741-1751. doi:10.1021/ acsearthspacechem.1c00083 
Perets, H. B., Lederhendler, A., Biham, O., Vidali, G., Li, L., Swords, S., et al. (2007). Molecular Hydrogen Formation on Amorphous Silicates under Interstellar Conditions. ApJ 661, L163-L166. doi:10.1086/518862

Potapov, A., Theulé, P., Jäger, C., and Henning, T. (2019). Evidence of Surface Catalytic Effect on Cosmic Dust Grain Analogs: The Ammonia and Carbon Dioxide Surface Reaction. ApJ 878, L20. doi:10.3847/2041-8213/ab2538

Powner, M. W., and Sutherland, J. D. (2011). Prebiotic Chemistry: A New Modus Operandi. Phil. Trans. R. Soc. B 366, 2870-2877. doi:10.1098/rstb.2011.0134

Qasim, D., Vlasak, L., Pital, A., Beckman, T., Mutanda, N., and Abbott-Lyon, H. (2017). Adsorption of Water, Methanol, and Formic Acid on Fe2NiP, a Meteoritic Mineral Analogue. J. Phys. Chem. C 121, 13645-13654. doi:10.1021/acs.jpcc.7b01312

Ross, J., and Arkin, A. P. (2009). Complex Systems: From Chemistry to Systems Biology. Proc. Natl. Acad. Sci. 106, 6433-6434. doi:10.1073/pnas.0903406106

Ruf, A., d'Hendecourt, L., and Schmitt-Kopplin, P. (2018). Data-Driven Astrochemistry: One Step Further within the Origin of Life Puzzle. Life 8, 18. doi:10.3390/life 8020018

Sandford, S. A., Bernstein, M. P., and Dworkin, J. P. (2010). Assessment of the Interstellar Processes Leading to Deuterium Enrichment in Meteoritic Organics. Meteorit. Planet. Sci. 36, 1117.

Shematovich, V. I. (2012). Formation of Complex Chemical Species in Astrochemistry (A Review). Sol. Syst. Res. 46, 391-407. doi:10.1134/ s0038094612060068

Sidhu, A., Peeters, E., Cami, J., and Knight, C. (2021). A Principal Component Analysis of Polycyclic Aromatic Hydrocarbon Emission in NGC 2023. Mon. Not. R. Astron. Soc. 500, 177.

Siegenfeld, A. F., and Bar-Yam, Y. (2020). An Introduction to Complex Systems Science and its Applications, 2020, 6105872. doi:10.1155/2020/6105872Complexity

Snyder, L. E., Buhl, D., Zuckerman, B., and Palmer, P. (1969). Microwave Detection of Interstellar Formaldehyde. Phys. Rev. Lett. 22, 679-681. doi:10.1103/ physrevlett.22.679

Stahl, P., Arenas, B. E., Domingos, S. R., Fuchs, G. W., Schnell, M., and Giesen, T. F. (2020). Laboratory Blueprints for Interstellar Searches of Aromatic Chiral Molecules: Rotational Signatures of Styrene Oxide. Phys. Chem. Chem. Phys. 22, 21474-21487. doi:10.1039/d0cp03523h

Sugahara, H., Takano, Y., Tachibana, S., Sugawara, I., Chikaraishi, Y., Ogawa, N. O., et al. (2019). Molecular and Isotopic Compositions of Nitrogen-Containing Organic Molecules Formed during UV-Irradiation of Simulated Interstellar Ice. Geochem. J. 53, 5-20. doi:10.2343/geochemj.2.0553

Suhasaria, T., and Mennella, V. (2021). Catalytic Role of Refractory Interstellar Grain Analogs on H2 Formation. Front. Astron. Space Sci. 8, 655883. doi:10.3389/fspas.2021.655883

Swings, P., and Rosenfeld, L. (1937). Considerations Regarding Interstellar Molecules. Astrophys. J. 86, 483-486. doi:10.1086/143880

Trakhtenberg, S., Naaman, R., Cohen, S. R., and Benjamin, I. (1997). Effect of the Substrate Morphology on the Structure of Adsorbed Ice. J. Phys. Chem. B 101, 5172-5176. doi:10.1021/jp9702412

Tsuge, M., Nguyen, T., Oba, Y., Hama, T., Kouchi, A., and Watanabe, N. (2020). UV-ray Irradiation Never Causes Amorphization of Crystalline CO2: A
Transmission Electron Microscopy Study. Chem. Phys. Lett. 760, 137999. doi:10.1016/j.cplett.2020.137999

van Dishoeck, E. F. (2014). Astrochemistry of Dust, Ice and Gas: Introduction and Overview. Faraday Discuss. 168, 9-47. doi:10.1039/c4fd00140k

Vinogradoff, V., Duvernay, F., Danger, G., Theulé, P., Borget, F., and Chiavassa, T. (2013). Formaldehyde and Methylamine Reactivity in Interstellar Ice Analogues as a Source of Molecular Complexity at Low Temperature. A\&A 549, A40. doi:10.1051/0004-6361/201219974

Wakelam, V., Bron, E., Cazaux, S., Dulieu, F., Gry, C., Guillard, P., et al. (2017). H 2 Formation on Interstellar Dust Grains: The Viewpoints of Theory, Experiments, Models and Observations. Mol. Astrophysics 9, 1-36. doi:10.1016/j.molap.2017.11.001

Weinreb, S., Barrett, A. H., Meeks, M. L., and Henry, J. C. (1963). Radio Observations of $\mathrm{OH}$ in the Interstellar Medium. Nature 200, 829-831. doi: $10.1038 / 200829 \mathrm{a} 0$

Whitesides, G. M., and Ismagilov, R. F. (1999). Complexity in Chemistry. Science 284, 89-92. doi:10.1126/science.284.5411.89

Widicus Weaver, S. L. (2019). Millimeterwave and Submillimeterwave Laboratory Spectroscopy in Support of Observational Astronomy. Annu. Rev. Astron. Astrophys. 57, 79-112. doi:10.1146/annurev-astro-091918-104438

Wollrab, E., Scherer, S., Aubriet, F., Carré, V., Carlomagno, T., Codutti, L., et al. (2016). Chemical Analysis of a "Miller-Type" Complex Prebiotic Broth. Orig. Life Evol. Biosph. 46, 149-169. doi:10.1007/s11084-015-9468-8

Yocum, K. M., Smith, H. H., Todd, E. W., Mora, L., Gerakines, P. A., Milam, S. N. et al. (2019). Millimeter/Submillimeter Spectroscopic Detection of Desorbed Ices: A New Technique in Laboratory Astrochemistry. J. Phys. Chem. A. 123, 8702-8708. doi:10.1021/acs.jpca.9b04587

Zakharenko, O., Ilyushin, V. V., Lewen, F., Müller, H. S. P., Schlemmer, S., Alekseev, E. A., et al. (2019). Rotational Spectroscopy of Methyl Mercaptan $\mathrm{CH} 332 \mathrm{SH}$ at Millimeter and Submillimeter Wavelengths. A\&A 629, A73. doi:10.1051/0004-6361/201935759

Conflict of Interest: The authors declare that the research was conducted in the absence of any commercial or financial relationships that could be construed as a potential conflict of interest.

Publisher's Note: All claims expressed in this article are solely those of the authors and do not necessarily represent those of their affiliated organizations or those of the publisher, the editors, and the reviewers. Any product that may be evaluated in this article, or claim that may be made by its manufacturer, is not guaranteed or endorsed by the publisher.

Copyright (C) 2021 Mason, Hailey, Mifsud and Urquhart. This is an open-access article distributed under the terms of the Creative Commons Attribution License (CC $B Y$ ). The use, distribution or reproduction in other forums is permitted, provided the original author(s) and the copyright owner(s) are credited and that the original publication in this journal is cited, in accordance with accepted academic practice. No use, distribution or reproduction is permitted which does not comply with these terms. 